Andrea Rapp*, Sabine Bartsch und Luise Borek

\title{
Aus der Mitte der Fächer, in die Mitte der Fächer: Studiengänge und Curricula - Digital Humanities in der universitären Lehre
}

DOI 10.1515/bfp-2016-0030

Zusammenfassung: Die universitäre Lehre gehört neben Forschung, Forschungsdaten und Infrastruktur $\mathrm{zu}$ den zentralen Säulen von DARIAH-DE. Die Aktivitäten richten sich auf drei Bereiche: auf die Konzeption und Durchführung von Lehreinheiten, Workshops und Tagungen, auf die Kartierung nationaler und internationaler DH-Studiengänge und Lehrangebote sowie auf die Organisation und Moderation der Diskussion um die Verortung und Entwicklung von DH-Curricula.

Schlüsselwörter: DH-Curricula; Lehrmaterialien; Course Registry

From the Centre of the Disciplines, Into the Centre of the Disciplines: Courses and Curricula - Digital Humanities in University Curricula

Abstract: University teaching forms one of the pillars of DARIAH-DE alongside research, research data, and infrastructure. Activities are targeting three areas: planning and conducting teaching units, workshops and conferences, a cartography of national and international $\mathrm{DH}$ curricula and courses, and the organization and moderation of discussions around the status and development of DH curricula.

Keywords: DH curricula; teaching materials; course registry

\section{Universitäre Lehre im Kontext von digitalen Forschungsinfrastruktu- ren: Das Beispiel DARIAH}

Wissenschaftliche Infrastrukturen wie Großgeräte, Forschungsbibliotheken oder auch digitale (Informations-)Infrastrukturen umfassen mehr als Gebäude, Sammlungen und (Zugang zu) Technologie, sie fungieren in aller Regel auch als „soziale Infrastrukturen“, ${ }^{1}$ in denen Kommunikation und Vermittlung eine entscheidende Rolle für ihre Etablierung und vor allem für ihre Nutzung spielen. Daher hat DARIAH - nicht zuletzt auch nach den Erfahrungen aus dem BMBF-geförderten Projekt TextGrid ${ }^{2}$ - vielfältige Lehraktivitäten, die weit über die Vermittlung der Infrastrukturangebote von DARIAH hinausgehen, von Anfang an als zentrale Säule in sein Konzept eingebunden. Lehre spielt auch im internationalen DARIAH-EU-Verbund eine wichtige Rolle: Das Virtual Competence Center 2 (VCC2) Research and Education ${ }^{3}$ hat das Project \#dariahTeach ins Leben gerufen, in dem gemeinsam mit ERASMUS+ und getragen von einer Vielzahl internationaler Partner multilinguale Open-Source-Lehrmaterialien entwickelt und verzeichnet werden. ${ }^{4}$

Einige der lehrbezogenen Aktivitäten von DARIAH-DE sollen im Folgenden herausgegriffen und in den Kontext der Etablierung einer digitalen Infrastruktur für die Geistes- und Kulturwissenschaften sowie der Professionalisierungs- und Institutionalisierungsentwicklungen der Digital Humanities - mit einem Fokus auf die Situation in Deutschland - gestellt werden.

1 Vgl. Wissenschaftsrat $(2012,19)$ und Wissenschaftsrat $(2011,25)$.

2 Rapp et al. (2014).

3 Zur Struktur der VCCS von DARIAH-EU: http://dariah.eu/activities. html.

4 http://dariah.eu/teach/index.php/2015/05/21/welcome-to-dariah teach.
*Kontaktperson: Prof. Dr. Andrea Rapp, rapp@linglit.tu-darmstadt.de Dr. Sabine Bartsch, bartsch@linglit.tu-darmstadt.de Luise Borek, M.A., borek@linglit.tu-darmstadt.de 


\section{Aktivitäten im Bereich universitäre Curricula}

Die Digital Humanities sind in Deutschland im Hinblick auf ihre universitäre Institutionalisierung einerseits noch ein junges Feld, andererseits in Nischen - eigentlich seit der Erfindung des Computers - seit Langem praktiziert, international bereits sehr gut aufgestellt und trotz aller Gemeinsamkeiten im Prinzip ebenso diversifiziert wie die Geistesund Kulturwissenschaften selbst. ${ }^{5}$ Daher richten sich die Aktivitäten von DARIAH sowohl auf die Verzeichnung und Durchführung von Lehrveranstaltungen als auch auf die Moderation und Organisation von Diskussionsprozessen rund um Lehre und Digitalität. Unter den erstgenannten Bereich fallen beispielsweise die Konzeption und die Ausrichtung von verschiedenen Veranstaltungen, Workshops oder Summerschools zu DARIAH-Kernthemen sowie die Beteiligung an curricularen Lehrveranstaltungen an den jeweiligen Projektstandorten und darüber hinaus. Damit eng verbunden ist die Erstellung eigener und die Verzeichnung weiterer Schulungsmaterialien. Zum letztgenannten gehören die Moderation und Mitgestaltung der Diskussionen um Curriculumsentwicklung in den Digital Humanities und ihre Verortung, ferner die Kartierung internationaler Lehrangebote. Diese Aktivitäten sind eng miteinander verzahnt und bilden gemeinsam mit den Themenkreisen Forschung, Forschungsdaten und technische Infrastruktur die Komponenten einer digitalen Infrastruktur, die die digital arbeitenden Geistes- und Kulturwissenschaften in Forschung und Lehre unterstützt und zugleich den internationalen Austausch befördert.

\subsection{Diskussion und Gestaltung von Studiengängen, Referenzcurriculum}

Die Einrichtung von Professuren mit entsprechender Denomination und die Akkreditierung von DH(nahen)-Studiengängen (derzeit zumeist auf Master-, seltener auf Bachelor-Niveau) sind ganz wesentliche Schritte der Etablierung der DH im universitären Ausbildungskanon und damit auch ihrer Institutionalisierung. Die Verortung und Ausrichtung der jeweiligen Studiengänge ist dabei Teil und Spiegel der Diskussionen um Definition und Selbstverständnis der Digital Humanities und ihrer Protagonisten. Folgendes Spektrum an (dezidierten) DH-Studiengängen zeichnet sich (ohne Anspruch auf Vollständigkeit) ab:

5 Vgl. z. B. Nyhan (2012) und Terras, Nyhan und Vanhoutte (2013).
- übergreifender DH-Master of Arts mit Beteiligung unterschiedlicher geisteswissenschaftlicher Disziplinen und Informatikanteilen (z. B. Würzburg, Planungen in Mainz),

- spezifischer, disziplinorientierter DH-Master of Arts mit Informatikanteilen (z. B. Darmstadt),

- übergreifender Master of Science-Studiengang aus geisteswissenschaftlichen, computerlinguistischen und informatischen Anteilen (z. B. Trier),

- Master of Science in der Angewandten Informatik (z. B. Göttingen),

- sog. „Bindestrich-Informatiken“ (z. B. Archäoinformatik Köln, Bamberg).

In Darmstadt, Göttingen und Würzburg sind ferner auch Bachelor-Angebote vorhanden, in Darmstadt ein fachspezifischer Bachelor of Arts Digital Philology, in Würzburg ein fachübergreifender Bachelor of Arts Digital Humanities und in Göttingen ein Zweifach-Bachelor of Science Informatik+Geistes-/Sozialwissenschaft. Allein diese Bandbreite zeigt, dass die Ausgestaltung der Studiengänge recht heterogen und der Austausch über gemeinsame curriculare Inhalte und Standards, aber auch Profilbildungen notwendig ist, um das Angebot weiter zu stabilisieren und die Studierenden und den wissenschaftlichen Nachwuchs auf dem Arbeitsmarkt und im universitären Umfeld konkurrenzfähig zu halten.

Aufbauend auf Vorarbeiten am Lehrstuhl für historisch-kulturwissenschaftliche Informationsverarbeitung (M. Thaller) an der Universität zu Köln und einer entsprechenden Arbeitsgruppe hat sich der DARIAH-Verbund insbesondere an der Bestandsaufnahme bestehender Angebote sowie der Organisation und Moderation des Diskussionsprozesses beteiligt. Zu diesen Kölner Vorarbeiten gehören u.a. die Organisation zweier Vorbereitungsworkshops mit breiter Beteiligung von DH-Akteuren und die Einrichtung und Betreuung einer weiter fortbestehenden und aktiven Mailingliste zu Curriculumsthemen mit rund 130 Teilnehmenden sowie die Sammlung einschlägiger Informationen zu Studiengängen. ${ }^{6}$ Wichtige Ergebnisse fasst der in den DARIAH Working Papers publizierte Bericht „DH studieren!“ von Patrick Sahle zusammen. ${ }^{7}$ Eine Fortsetzung und Ausweitung der Diskussion erfolgte seit 2009 durch die Verortung der Arbeitsgruppe Referenzcurriculum Digital Humanities als AG innerhalb des 2013 ge-

6 Zur Dokumentation der Vorbereitungstreffen 2009 und 2010: http://www.cceh.uni-koeln.de/events/DH-curricular und http://www. cceh.uni-koeln.de/events/DH-curricular-II; Mailingliste: cceh-dhcurri cular@uni-koeln.de.

7 Sahle (2013). 
gründeten Fachverbands Digital Humanities im deutschsprachigen Raum. ${ }^{8}$ In einem dynamischen Feld wie den Digital Humanities muss ein ,Referenzcurriculum “ die Gratwanderung zwischen Verbindlichkeit und Standardisierung einerseits und Flexibilität und Gestaltungsraum meistern. Die DHd-Arbeitsgruppe versteht daher den Begriff ,Referenzcurriculums“ im Sinne einer ,Referenzarchitektur', die ein Modell beschreibt, das Vergleichbarkeit ermöglicht. Die AG ist offen strukturiert, eine aktive Beteiligung der Community erwünscht. Der Stand der Diskussionen und Entwicklungen wurde bei der DHd-Jahrestagung 2015 in Graz als Broschüre unter dem Titel „Digital Humanities als Beruf. Fortschritte auf dem Weg zu einem Curriculum"9 vorgelegt.

Aktuelle Fragen drehen sich beispielsweise darum, in welcher Fakultät die DH zu verorten sind, welchen Stellenwert und Gewichtung Geisteswissenschaft und Informatik jeweils haben, welche Informatikinhalte, Auszeichnungsund Programmiersprachen unterrichtet werden und wie sie mit geisteswissenschaftlichen Lehrplaninhalten kombiniert werden sollen. Diese Fragen lassen sich auch auf internationaler Ebene weiterverfolgen,${ }^{10}$ wozu insbesondere DARIAH-EU einen wichtigen Beitrag leisten kann.

Diese Diskussionen speisen sich auch aus den umfangreichen praktischen Lehraktivitäten, die DARIAH-Mitglieder sowohl im Rahmen von etablierten DH-Studiengängen als auch in unterschiedlichen Workshopformaten durchführen und über die im folgenden Abschnitt informiert werden soll.

\subsection{Workshopformate: Fort- und Weiterbildung}

Neben der Diskussion um Studieninhalte und -formate wurde eine breite Palette an forschungs-, methoden- und zielgruppenspezifischen Workshops und Seminaren durchgeführt $\mathrm{t}^{11}$ und Beteiligungen an bereits in der Community etablierten Summerschools sowie weiteren gängigen wissenschaftlichen Tagungen und Veranstaltungen ${ }^{12}$ ini-

8 http://dig-hum.de/arbeitsgruppe-referenzcurriculum-digital-huma nities.

9 Digital Humanities als Beruf (2015).

10 Hirsch (2012).

$11 \mathrm{https} / / /$ de.dariah.eu/veranstaltungen.

12 Es wurden z. B. durchgängig Workshops auf den großen nationalen und internationalen DH-Konferenzen des DHd-Verbands (http:// www.dig-hum.de) bzw. der ADHO (http://adho.org/conference) platziert; darüber hinaus war der Verbund auch auf den Tagungen weiterer geisteswissenschaftlicher Fachverbände, dem Bibliothekartag sowie spezifischen Fachtagungen präsent. tiiert. Die unterschiedlichen Veranstaltungstypen richten sich gezielt an bestimmte Zielgruppen und verfolgen jeweils eigene methodische Konzepte. Expertenseminare und Methodenworkshops sind sowohl ihrer Zielgruppe als auch ihrer Inhalte nach voneinander abgegrenzt: Methodenworkshops richten sich in erster Linie an Nachwuchswissenschaftler und ,Novizen“ in den Digital Humanities, während Expertenseminare die kritische Auseinandersetzung mit Methoden adressieren, nicht die Weiterentwicklung von Tools. Dozentenworkshops wiederum dienen der Fortbildung und dem Austausch von Lehrenden.

Expertenseminare sollen demnach etablierte Experten aus den Digital Humanities und Fachexperten der Geistesund Kulturwissenschaften möglichst in Verbindung mit einem konkreten Forschungsvorhaben in einen Austausch bringen und so eine intensive Auseinandersetzung mit den Möglichkeiten, Grenzen, vor allem aber dem Entwicklungspotential bestimmter DH-Verfahren und -Tools in Gang bringen. Die Diskussion zweier Gruppen ,auf Augenhöhe“ erlaubt wichtige Erkenntnisse und Fortschritte auf beiden Seiten: Fachexperten können ein tieferes Methodenverständnis sowie konkrete Kompetenzen in der Anwendung digitaler Tools und Methoden erreichen, um auf diese Weise ihr Methodenrepertoire zu erweitern, während DH-Experten Erfahrungen sammeln können, welche Bedarfe im Hinblick auf Tools und Infrastruktur aus den Geistes- und Kulturwissenschaften artikuliert werden. Internationale Gruppen trafen sich beispielsweise $\mathrm{zu}$ den Themen ,A Controlled Vocabulary for Historical Place Types‘ (Mainz 2013) oder ,Intelligent Annotations‘ (Köln 2015). Die Expertenseminare haben sich als sehr gut geeignet erwiesen, spezifische Netzwerke und Kooperationen aufzubauen. Ergebnisse aus diesen Seminaren können unmittelbar in die DARIAH-Infrastruktur zurückfließen.

Methodenworkshops sind prinzipiell vergleichbar angelegt in ihrem Ziel, eine Begegnung auf Augenhöhe zu ermöglichen, inhaltlich sind sie auf Forschungsprobleme und -methoden fokussiert, so dass ein konkretes Forschungsproblem und die digitalen Methoden, die für die Bearbeitung der jeweiligen Forschungsfragen Beiträge leisten können, zusammengebracht werden. Die adressierten thematischen Perspektiven sind dabei nicht disziplinspezifisch, sondern disziplinübergreifend. Neben dem anwendungsorientierten Praxisbezug steht vor allem der Mehrwert, der durch den Einsatz digitaler Methoden in den Geisteswissenschaften erreicht werden kann, im Fokus der Diskussion. Auf diese Weise wird in den Fachwissenschaften eine Reflexion über die Entwicklung und Leistungsfähigkeit einer Forschungsmethode sowie ihre Transformation und Erweiterung im Kontext der Digitalität befördert. In den Digital Humanities können Stärken und Schwächen 
der Verfahren in Bezug auf konkrete Forschungsprobleme analysiert und aufgedeckt werden. Die Bandbreite der adressierten Themen war enorm vielfältig und reichte beispielsweise von ,Natural Language Processing für Literaturwissenschaftler ' (Würzburg 2015) über ,Lizenzfragen für Forschungsdaten“ (DAI Berlin 2013) bis zur ,App-Entwicklung (Darmstadt 2015). Aus einigen Workshops sind auch DARIAH Working Papers hervorgegangen.

Die von erfahrenen DH-Lehrenden geleiteten Dozentenworkshops sollten auf den Bedarf an Fortbildungsmaßnahmen reagieren, der durch die Verfügbarkeit digitaler Forschungsdaten, rasch wechselnde Technologiezyklen und die Ausbreitung und Akzeptanz der Digital Humanities stark gestiegen ist. Diese praxisbezogenen Workshops waren auf einen eher homogenen Teilnehmerkreis ausgerichtet, in dem der Einsatz von digitalen Methoden und Tools in der universitären Lehre besprochen wurde. Auf diese Weise sollten neue Kompetenzen in die eigene Lehre einfließen und die Digital Humanities in die Mitte der Fächer gebracht werden. Nach den beiden Dozentenworkshops ,Strategien für cooperatives Lehren mit WIKIs in den Geistes- und Kulturwissenschaften' (Würzburg 2012) und ,Curricularentwicklungen in den Digital Humanities‘ (Köln 2013) wird dieses Format derzeit nicht fortgeführt, da die übrigen Formate genügend andere Möglichkeiten der gezielten Vermittlung bieten.

Summerschools schließlich sind eine weithin etablierte Möglichkeit der gezielten Nachwuchsförderung und Weiterbildung und werden häufig auch im Kontext universitärer Studiengänge als curricularer Leistungsnachweis anerkannt. Da sich für die $\mathrm{DH}$ ein breites und vielfältiges nationales wie internationales Angebot an Summerschools etabliert hat, konzentrieren sich die DARIAH-Aktivitäten hier auf Beteiligungen an solchen bestehenden Summerschools, nicht auf die Einrichtung einer weiteren eigenen School. ${ }^{13}$ Dies hat den weiteren Effekt der intensiveren Vernetzung und Dissemination in die Community hinein.

\subsection{Schulungsmaterialien: Kooperation mit OERCommons, neue Vermittlungsformate und Medien}

DARIAH-DE und seine assoziierten Projekte erstellen einerseits eigene Schulungsmaterialien, z. B. zu TEI oder zur

$13 \mathrm{Zu}$ nennen sind hier z. B. die EDIROM Summerschool an der Universität Paderborn (http://ess.upb.de) oder die European Summer University in Digital Humanities „Culture \& Technology“ an der Universität Leipzig (http://www.culingtec.uni-leipzig.de/ESU_C_T). (quantitativen) Kodikologie, anderseits wird eine entsprechende Sammlung zu Verfahren, Methoden und Themen der Digital Humanities angelegt. Sie umfasst mit dem Stand Februar 2016228 Angebote von freien Schulungsund Lehrmaterialien von internationalen Wissenschaftlern. Sämtliche von DARIAH-DE publizierten Schulungsmaterialen werden unter der Creative Commons Lizenz CCBY 4.0 zur Verfügung gestellt, ${ }^{14}$ die eine freie Nutzung unter Nennung der Urheber in sämtlichen Kontexten erlaubt. Auch weniger frei lizensierte Materialien externer Anbieter werden verzeichnet, um einen Überblick über das Feld mit einem zentralen Nachweis zu bieten, der sowohl über das DARIAH-Portal wie auch über eine OERCommons-Seite durchsucht werden kann. ${ }^{15}$

Die erstellten und verzeichneten Materialien sind disparat im Hinblick auf ihre Sprachen, ihre Medien und die didaktische Durchdringung: Neben didaktisch aufbereitetem Material wie den TEI- oder TextGrid-Tutorials finden sich beispielsweise auch Vorlesungsaufzeichnungen und Informationsmaterial zu spezifischen Themen oder ToolDokumentationen (z.B. zu DigiVoy). ${ }^{16}$ Eine Kategorisierung der Materialien nach curricularen Zuordnungen ermöglicht eine rasche Orientierung im Angebot, derzeit werden die folgenden Bereiche abgedeckt: Computerlinguistik/Sprachverarbeitung, Digital Humanities, Digitale Bibliotheken und Datenbanken, Media, Semantic Technologies, Software Engineering, Technische Anwendung sowie Technische Grundlagen.

Das DH-Dashboard erlaubt einen raschen und effizienten Zugriff auf Materialien für bereits informierte Nutzende. ${ }^{17}$ Social Media-Kanäle wie Twitter und ein DHd-Kanal ${ }^{18}$ bereichern die Vermittlungs-Palette um eine weitere Facette. Mit ihnen erschließt DARIAH auch neue Adressatenkreise und erreicht rasch und effizient die in diesem Bereich sehr aktive und professionell agierende DH-Community. Auf dem YouTube-Kanal können Veranstaltungsmitschnitte, Vorträge und Vorlesungen, Info-Clips für unterschiedliche Adressatengruppen sowie Videotutorials zu DH-Themen und zu verschiedenen Tools und Diensten gebündelt werden. Explainity Clips mit ihrer Komplexitätsreduktion bieten einen niedrigschwelligen Zugang zu zentralen DH-

14 Creative Commons Lizenz; Version 4.0: https://creativecommons. org/licenses/by/4.0.

15 Vgl. Neuschäfer (2015).

16 https://de.dariah.eu/digivoy.

$17 \mathrm{http} / / /$ dhdashboard.de.

18 Der Twitteraccount von @DARIAHde hat derzeit weit über 1500 Follower und erreicht gemeinsam mit den Accounts der übrigen europäischen DARIAH-Projekte eine breite Community durch Microblogging: https://twitter.com/dariahde?lang=de; der YouTube-Kanal findet sich hier: https://www.youtube.com/user/dhdkanal. 
Themen wie ,Kollaboration“ oder ,Datensicherheit‘ auf einfachste Weise und ermöglichen dadurch eine weitere Öffnung und sprechen nicht zuletzt Schüler an.

Schließlich stellt DARIAH-DE die nach Themenfeldern gegliederte Bibliographie Doing Digital Humanities mit DHLiteratur von Einführungen bis zu vertiefender Forschungsliteratur zur Verfügung, die über die TaDiRAHTaxonomie erschlossen ist (s.u.). Sie wird kollaborativ über eine Zotero-Group erstellt und ist über das DARIAHPortal einsehbar, kann aber auch über Zotero genutzt werden, wo erweiterte Funktionen zur Verfügung stehen. ${ }^{19}$

\section{Die TaDiRAH-Taxonomie}

TaDiRAH steht für Taxonomy of Digital Research Activities in the Humanities und ist über das GitHub-Repository frei zugänglich. ${ }^{20}$ Sie basiert auf verschiedenen Vorarbeiten und greift einige methodologische Überlegungen und Konzepte auf. Zum einen bot der DARIAH Report ,Verfahren der Digital Humanities in den Geistes- und Kulturwissenschaften ${ }^{21}$ die Gelegenheit, einen Überblick über aktuelle Verfahren zu schaffen. Zum anderen regten die Kooperationen mit dem Network for Digital Methods in the Arts and Humanities (NeDiMAH), zu dessen Zielen die Entwicklung einer Ontologie digitaler Forschungsmethoden gehörte, sowie mit DiRT, ${ }^{22}$ das mit seinem Directory eine Registry für digitale Tools anbietet, gemeinsame und weiterführende Aktivitäten zur Erstellung, Pflege und Nutzung einer Taxonomie von DH-Verfahren an. Damit entsteht ein komplexes Modell für die Beziehung von Forschungsfeldern, Methoden, Institutionen sowie Forschungsdaten und -ergebnissen, das mit seiner Formalisierung des Verständnis‘ der DH nicht nur zum Ausbau der digitalen Forschungsinfrastruktur, sondern auch zur Reflexion und Diskussion um die Verortung der DH sowie ihre weitere Vernetzung beiträgt.

Die Taxonomie bezieht methodologische Überlegungen von Willard McCarty und Harold Short ${ }^{23}$ sowie von John Unsworth ${ }^{24}$ mit ein: McCarty/Short entwickeln die Idee der „methodological commons“ und einem darauf basierenden „mapping“ mit den „commons“ als Zentrum,

19 https://www.zotero.org/groups/doing_digital_humanities_-_a_d ariah_bibliography.

20 https://github.com/dhtaxonomy/TaDiRAH; s. zu TaDiRAH auch Borek et al. (2016) und Borek et al. (2014).

21 Reiche et al. (2014).

22 http://www.nedimah.eu und http://dirtdirectory.org.

23 McCarty und Short (2002).

24 Unsworth (2000). um ein Modell digitaler Methoden zu erhalten, das von den Anwendungsfeldern geteilt wird und als Schnittstelle zwischen Disziplingruppen und Lehrbereichen dient. Die TaDiRAH-Kategorie ,object ${ }^{\text {‘ }}$ ist an den ,data types ${ }^{\text {‘ von }}$ McCarty/Short orientiert. John Unsworth spricht von „Scholarly Primitives“, die für ihn basale Aktivitäten im Forschungsprozess sind, die Forschungsfeldern und Medientypen gemeinsam sind und diese unabhängig von Disziplin und Methoden verbinden. Unsworths Basis-Kategorien Discovering, Annotating, Comparing, Referring, Sampling, Illustrating und Representing werden in TaDiRAH aufgegriffen.

Die Taxonomie ist beispielsweise zur Erschließung der DARIAH-Bibliographie im Einsatz und erfährt eine multilinguale Erweiterung durch ihre aktuellen Übersetzungen ins Deutsche, Spanische und Serbische.

\section{Course Registry}

Die Digital Humanities Course Registry ist eine weitere Aktivität des DARIAH-EU Virtual Competence Centers 2 (VCC2) Research and Education, mit der die DH-Lehrangebote in Europa kartiert werden. ${ }^{25}$ Dabei können Angebote unterschiedlichster Art verzeichnet werden: von einer kurzen Lehreinheit oder einem DH-Kursanteil im Kontext geisteswissenschaftlicher oder informatischer Seminarangebote über einzelne DH-Module in „traditionellen“ Studiengängen bis hin zu eigenständigen DH-Studiengängen. Die Registry wird derzeit in Darmstadt betreut, kann jedoch von registrierten Nutzenden selbständig gefüllt werden. Hauptziel ist hier, Studierenden, Lehrenden, Forschenden und wissenschaftlichen Institutionen eine Möglichkeit zur Identifikation von Lehr-, Studien- und Fortbildungsangeboten zu geben, aber auch die Vernetzung und den Austausch unter den Anbietenden zu erleichtern. Nicht zuletzt bringt ihnen die Verzeichnung eine erhöhte internationale Sichtbarkeit.

\section{Ausblick: Auf dem Weg zu einer digitalen Didaktik}

Wie der kurze Überblick am Beispiel DARIAH zeigen konnte, bilden derzeit Überlegungen $\mathrm{zu}$ Unterrichtsinhalten und -formen bzw. die Curriculumsentwicklung für (neue) Digital-Humanities-Studiengänge das Zentrum der Diskus-

25 https://dh-registry.de.dariah.eu. 
sion. Sie konzentrieren sich ferner weitgehend auf Szenarien der universitären Wissensvermittlung, vor allem werden Konzepte und Materialien für DH-Lehrende entwickelt. Konzepte, mit denen die Studierenden selbst zum fachdidaktisch-pädagogischen Arbeiten mit digitalem Bezug befähigt werden, sind noch Desiderat, Ansätze der DH zu schulischen und außerakademischen Lehr-Lern-Szenarien ebenfalls. Hier gilt es, Anknüpfungspunkte an andere Fachrichtungen und ihre Didaktik mit Ausrichtung auf das Digitale zu entwickeln. Impulse können beispielsweise Ansätze aus der Didaktik der Informatik, ${ }^{26}$ aus dem eLearning $^{27}$ und der Medienpädagogik/-didaktik ${ }^{28}$ geben. Es wird darüber hinaus danach zu fragen sein, was die DH zur Neu- und Weiterentwicklung fachdidaktischer Konzepte und Methoden mit Bezug zu digitalen Inhalten und Kulturtechniken beitragen können, damit didaktische Aspekte des Digitalen vor allem als Gegenstand des Wissenstransfers an Studierende thematisiert und entwickelt werden können. Didaktisch-pädagogische Vermittlungsfertigkeiten sind wichtige Kompetenzen für DH-Absolventen - ob in der universitären Lehre, in der Forschung oder in Unternehmen: Die Vermittlung des Mehrwerts informationstechnisch gestützten wissenschaftlichen Arbeitens, der besonderen Merkmale digitaler Untersuchungsgegenstände, der Methoden und Verfahren der DH und ihrer informatischen und geisteswissenschaftlichen Konzepte - auch gerichtet an weniger technikaffine oder weniger geisteswissenschaftlich arbeitende Zielgruppen sind Kernaufgaben von Digital Humanists. Aber auch für Lehramtsstudierende ist Didaktik im Hinblick auf digitale Inhalte, Anwendungen und Methoden wichtig, ebenso wie für Studierende von nicht explizit auf das Digitale ausgerichteten Studiengängen, da auch hier digitale Untersuchungsgegenstände und Kulturtechniken an Bedeutung gewinnen. Die großen Entwicklungs-, Bündelungs- und Vermittlungsleistungen einer europäischen digitalen Forschungsinfrastruktur, wie sie mit DARIAH-EU und auch CLARIN-EU ${ }^{29}$ ermöglicht werden, bilden zuallererst die Basis für solche weiteren Bestrebungen in Bildung und Ausbildung.

26 Schubert und Schwill (2011).

27 Arnold et al. (2013).

28 Frederking, Krommer und Maiwald (2012).

29 CLARIN = Common Language and Resources and Technology Infrastructure, s. http://clarin.eu.

\section{Literaturverzeichnis}

Arnold, Patricia; Kilian, Lars; Thillosen, Anne; Zimmer, Gerhard (2013): Handbuch E-Learning. Lehren und Lernen mit digitalen Medien. 3., aktualisierte Aufl., Bielefeld: wbv.

Borek, Luise; Dombrowski, Quinn; Perkins, Jody; Schöch, Christof (2016): TaDiRAH - A Case Study in Pragmatic Classification. In: Digital Humanities Quarterly, 10 (1). Verfügbar unter http:// www.digitalhumanities.org/dhq/vol/10/1/000235/000235. html.

Borek, Luise; Perkins, Jody; Dombrowski, Quinn; Schöch, Christof (2014): Building bridges to the future of a distributed network: From DiRT categories to TaDiRAH. A methods taxonomy for digital humanities. Proc. International Conference on Dublin Core and Metadata Applications.

Digital Humanities als Beruf (2015): Fortschritte auf dem Weg zu einem Curriculum. Akten der DHd-Arbeitsgruppe „Referenzcurriculum Digital Humanities“. Vorgelegt auf der Jahrestagung 2015 Graz, 24. bis 27. Februar 2015. Redaktion: Zoe Schubert. Verfügbar unter http://www.dh-curricula.org/index.php?id=mater ials; https://www.digitalhumanities.tu-darmstadt.de/index.ph p?id=materials.

Frederking, Volker; Krommer, Axel; Maiwald, Klaus (2012): Mediendidaktik Deutsch. Eine Einführung. 2., neu bearbeitete und erweiterte Aufl., Berlin: Schmidt.

Hirsch, Brett D. (Hg.) (2012): Digital Humanities Pedagogy: Practices, Principles and Politics. Cambridge: Open Book Publ.

McCarty, Willard; Short, Harold (2002): Mapping the Field. In: EADH. Verfügbar unter http://eadh.org/publications/mapping-field.

Neuschäfer, Markus (2015): OER Commons: Offene Lehrmaterialien für die Digital Humanities. In: DHd-Blog, 22.7.2015. Verfügbar unter http://dhd-blog.org/?p=5424.

Nyhan, Julianne (Hg.) (2012): Hidden Histories: Computing and the Humanities c. 1965-1985. In: Digital Humanities Quaterly, 6 (3). Verfügbar unter http://www.digitalhumanities.org/dhq/vol/6/3 /index.html.

Rapp, Andrea; Schmid, Oliver; Vanscheidt, Philipp; Krause, Celia (2014): Erfahrungen aus den Nutzerschulungen. Empfehlungen für die weitere Ausgestaltung des Konzepts (Report R 3.3.1). Verfügbar unter https://textgrid.de/fileadmin/TextGrid/reports/Te xtGrid_Report_3_3_1_Final.pdf.

Reiche, Ruth; Becker, Rainer; Bender, Michael; Munson, Matthew; Schmunk, Stefan; Schöch, Christof (2014): Verfahren der Digital Humanities in den Geistes- und Kulturwissenschaften. DARIAHDE Working Papers 4. Göttingen: DARIAH-DE. Verfügbar unter urn:nbn:de:gbv:7-dariah-2014-2-6.

Sahle, Patrick (2013): DH studieren! Auf dem Weg zu einem Kern- und Referenzcurriculum der Digital Humanities. DARIAH-DE Working Papers 1. Göttingen: DARIAH-DE. Verfügbar unter urn:nbn:de: gbv:7-dariah-2013-1-5.

Schubert, Sigrid; Schwill, Andreas (2011): Didaktik der Informatik. 2. Aufl., Heidelberg: Spektrum Akademischer Verlag.

Terras, Melissa; Nyhan, Julianne; Vanhoutte, Edward (Hg.) (2013): Defining Digital Humanities: A Reader. Farnham: Ashgate.

Unsworth, John (2000): Scholarly Primitives: What Methods Do Humanities Researchers Have in Common, and How Might Our Tools Reflect This? London: King's College. Verfügbar unter http://people.brandeis.edu/ unsworth/Kings.5-00/primitives. html. 
Wissenschaftsrat (2011): Übergreifende Empfehlungen zu Informationsinfrastrukturen. Drs. 10466-11, Berlin 28.01.2011. Verfügbar unter http://www.wissenschaftsrat.de/download/archiv/10466 -11 .pdf.

Wissenschaftsrat (2012): Empfehlungen zur Weiterentwicklung der wissenschaftlichen Informationsinfrastrukturen in Deutschland bis 2020. Drs. 2359-12 Berlin 13.07.2012. Verfügbar unter htt p://www.wissenschaftsrat.de/download/archiv/2359-12.pdf.

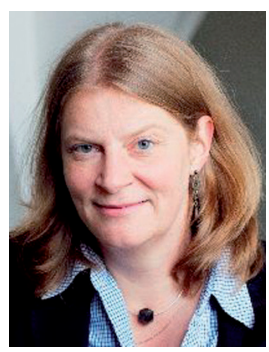

Prof. Dr. Andrea Rapp

Technische Universität Darmstadt Institut für Sprach- und Literaturwissenschaft

Dolivostr. 15

D-64293 Darmstadt

rapp@linglit.tu-darmstadt.de

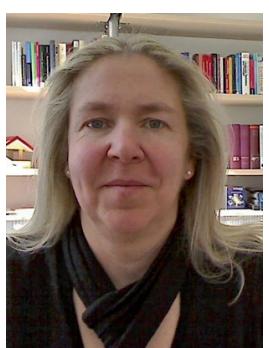

\section{Dr. Sabine Bartsch}

Technische Universität Darmstadt Institut für Sprach- und

Literaturwissenschaft

Dolivostr. 15

D-64293 Darmstadt

bartsch@linglit.tu-darmstadt.de

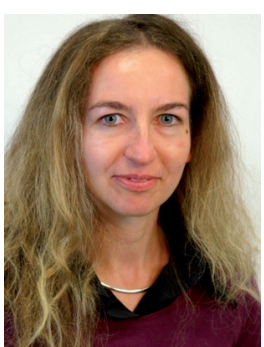

\section{Luise Borek, M. A}

Technische Universität Darmstadt Institut für Sprach- und

Literaturwissenschaft

Dolivostr. 15

D-64293 Darmstadt borek@linglit.tu-darmstadt.de 